\title{
Zur Bewertung von Wandelbarkeit in der Intralogistik
}

\author{
Dipl.-Ing. Jan Robert Nopper, Univ.-Prof. Dr. Michael ten Hompel \\ Fraunhofer-Institut für Materialfluss und Logistik \\ Dortmund
}

\begin{abstract}
Aufgrund der gestiegenen Komplexität und Dynamik der Einsatzgebiete spielen Flexibilität und Wandelbarkeit in der Logistik eine immer wichtigere Rolle. Dabei sind insbesondere eine einfache Erweiterbarkeit und Modifizierbarkeit bestehender Anlagen bei sich ändernden Umweltbedingungen wichtig. Während zusätzliche (Investitions-) Kosten wandelbarerer Systeme oft vergleichsweise einfach zu bewerten sind, ist eine quantitative Würdigung der Vorteile wandelbarer Systeme bis heute schwierig.
\end{abstract}

Der vorliegende Beitrag schlägt ein analytisches Berechnungsverfahren zur quantitativen Beurteilung von Wandelbarkeit in der Intralogistik vor. Dieses berücksichtigt den gesamten Lebenszyklus des Materialflusssystems und quantifiziert die mit einer erhöhten Wandelbarkeit verbundenen Effizienzgewinne in Geldeinheiten. Das Verfahren erlaubt den Umgang mit einer unsicheren Zukunft. Grundsätzlich werden für die Bewertung die zu vergleichenden Materialflusssysteme über die durch Wandelbarkeit beeinflussten Auszahlungen im Lebenszyklus modelliert. Für alle realistischen Entwicklungen des zukünftigen Bedarfs werden anschließend die optimalen Anpassungsschritte bestimmt. Dies geschieht mit Hilfe einer dynamischen Optimierung unter Ausnutzung des Bellmann'schen Optimalitätsprinzips. Leicht wandelbare Systeme zeigen dabei kleinere und dafür häufigere optimale Anpassungen als schwer wandelbare Systeme. Dadurch ergeben sich in der Regel Vorteile hinsichtlich der Betriebskosten, der Investitionskosten und es fallen geringere Kosten aufgrund von Unterdeckungen an. Darüber hinaus ergibt sich im Falle einer unsicheren Zukunft ein zusätzlicher Vorteil, da der Informationsgewinn im Zeitverlauf besser berücksichtigt werden kann. Der Einsatz des Verfahrens wird anhand eines realistischen Anwendungsbeispiels erläutert.

Dieser Beitrag ist eine Zusammenfassung von Ergebnissen der Dissertation des ersten Autors, die vom zweiten Autor betreut wurde.

\section{$1 \quad$ Einführung}

Aufgrund der gestiegenen Komplexität und Dynamik der Einsatzgebiete spielen Flexibilität und Wandelbarkeit in der Logistik eine immer wichtigere Rolle. Dabei ist vor allem eine einfache Erweiterbarkeit und Modifizierbarkeit bestehender Anlagen bei sich ändernden Umweltbedingungen wichtig. Während zusätzliche (Investitions-) Kosten wandelbarerer Systeme oft vergleichsweise einfach zu bewerten sind, ist eine quantitative Würdigung der Vorteile wandelbarer Systeme in der Regel schwierig [Sla83], [SeS90]. 
Technisch kann eine erhöhte Wandelbarkeit von Materialflusssystemen unter anderem über eine dezentrale Steuerung erreicht werden, insbesondere in Verbindung mit RFID. Über eine konsequente Modularisierung der Förder- und Steuerungstechnik lassen sich so selbstorganisierte Materialflusssysteme realisieren - das "Internet der Dinge in der Intralogistik" (vgl. hierzu z.B. [BtH07, [GCK08a], [GCK08b], [GCK08c], [May09], [tH06], [tHFR08]). Eine Kopplung von Modulen zum jeweils benötigten System verspricht eine hohe Flexibilität bezüglich Erweiterungen und Änderungen, die in der Intralogistik als Wandelbarkeit bezeichnet wird. Selbstorganisierte Materialflusssysteme werden dabei aus standardisierten fördertechnischen Modulen aufgebaut, die jeweils einen eigenständigen Dienst anbieten, beispielsweise den Transport von einer Quelle zu einer Senke oder die Pufferung für einen gewissen Zeitraum. Jedes Modul umfasst neben der Fördertechnik und den Antrieben auch die zur Erfüllung des Dienstes notwendige Steuerung. Diese Module können dann zum jeweils benötigten Materialflusssystem kombiniert werden. Das Transportgut steuert dieses System dezentral, indem es die angebotenen Dienste entsprechend seiner Ziele anfordert und sich mit anderen transportierten Einheiten koordiniert. Selbstorganisierte Materialflusssysteme wurden in Forschung und Praxis bereits realisiert, unter anderem in Versuchsanlagen des Lehrstuhls für Förder- und Lagerwesen der Technischen Universität Dortmund und des Lehrstuhl Fördertechnik Materialfluss Logistik der Technischen Universität München sowie dem THINGtelligence Konzept der Firma Lanfer CARGO-Technik.

Aber auch andere Ansätze zielen in Richtung einer Erhöhung der Wandelbarkeit von Materialflusssystemen, z.B. das Konzept Hub2Move des Fraunhofer-Instituts für Materialfluss und Logistik in Dortmund. Ziel hierbei ist die Gestaltung von flexiblen Logistikknoten, die je nach Bedarf aufgebaut, erweitert oder versetzt werden können.

Allerdings ist die Einführung von wandelbaren Materialflusssystemen auch mit Kosten verbunden, beispielsweise höheren Betriebskosten aufgrund verwendeter RFID-Tags oder höheren Investitionskosten durch die notwendige Erweiterbarkeit der Anlage. Bisher existiert kein Verfahren zur Quantifizierung von Wandelbarkeit in der Intralogistik über den Lebenszyklus. Daher kann die wirtschaftliche Effizienz selbstorganisierter Materialflusssysteme bisher nicht umfassend bewertet werden. Ziel des vorliegenden Beitrags ist es, eine Methodik zur Bewertung von Wandelbarkeit in der Intralogistik vorzustellen, die den gesamten Lebenszyklus des Materialflusssystems berücksichtigt und die mit einer erhöhten Wandelbarkeit verbundenen Effizienzgewinne monetär quantifiziert.

\section{Zur Bewertung von Flexibilität und Wandelbarkeit}

Unter Wandelbarkeit versteht man in der Intralogistik die Erweiterbarkeit und Veränderbarkeit des Materialflusssystems über die beim Aufbau des Systems gesetzten Grenzen hinaus [GHW02]. Damit unterscheidet sie sich von der Flexibilität, die die Änderung eines Systems innerhalb der beim Aufbau vorgedachten Grenzen beschreibt. Der Begriff Wandelbarkeit entspricht somit dem englischen Begriff (C) 2010 Logistics Journal : Proceedings - ISSN 2192-9084 
expansion flexibility. Wandelbarkeit in der Logistik muss dabei grundsätzlich entlang der Dimensionen Fördergut, Layout und Durchsatz betrachtet werden [GHW02]. Nur innerhalb dieser Dimensionen können Erweiterungen oder Veränderungen des Systems vorgenommen werden. Dabei muss nicht in jeder Situation jede der drei Dimensionen relevant sein.

Allerdings ist eine Bewertung von Wandelbarkeit heute schwierig - insbesondere in der Intralogistik gibt es hierfür bisher nur wenige Ansätze. Alle vorhandenen Ansätze lassen sich in direkte und indirekte Verfahren unterscheiden (vgl. hierzu auch [SeS90] und [Möl08]). Die direkten Verfahren definieren eine direkte Kenngröße für Wandelbarkeit und messen diese in der sich ergebenden Einheit. Im zweiten Schritt lassen sich die direkten Verfahren weiter unterscheiden in qualitative und quantitative Ansätze. Erstere umfassen hauptsächlich Ansätze, die auf einer gewichteten Punktbewertung oder der Nutzwertanalyse beruhen. Quantitative, direkte Ansätze beinhalten zum Beispiel direkte Verhältniskennzahlen wie das Verhältnis der mit einer Anlage erreichbaren Zustände zu allen theoretisch möglichen Zuständen.

Für die direkten Bewertungsverfahren spricht, dass sie in der Regel gut verständlich und einfach anzuwenden sind. Außerdem benötigen sie oft nur wenige Eingabedaten. Allerdings haben sie einen geringen praktischen Bezug: In der Regel möchte ein Entscheidungsträger die zusätzlichen (Investitions-) Kosten der Wandelbarkeit mit einem Nutzen vergleichen, der in gleichen - das heißt monetären - Einheiten gemessen wird.

Diese Eigenschaft bieten die indirekten Verfahren, welche Wandelbarkeit mit Hilfe einer monetären Ersatzgröße bewerten. Grundlage kann die Kapitalwertmethode sein, die jedoch wegen der dynamischen Natur von Wandelbarkeit mit anderen Methoden kombiniert werden muss. Möglich sind hier beispielsweise qualitative Methoden, Methoden des Operations Research oder das Entscheidungsbaumverfahren. Weiterhin kann Wandelbarkeit auch mit Hilfe von Realoptionen bewertet werden. Dabei wird die Wandelbarkeit eines Materialflusssystems als Option für eine zukünftige Änderung oder Erweiterung betrachtet. Über einen Vergleich mit bekannten und an Finanzmärkten gehandelten Werten kann so mit Hilfe der Optionspreistheorie ein Wert dieser Optionen berechnet werden. Vorteilhaft an einer Bewertung mit Realoptionen sind die stringente Methodik und die Verwendung risikoadjustierter Zinssätze. Allerdings ist sie relativ unbekannt und beinhaltet eine komplexe Berechnung, die ein intuitives Verständnis und den Umgang mit dem Verfahren erschwert. Außerdem ist es in der Praxis oft schwierig, einen geeigneten Vergleichswert $z u$ finden. Dagegen ist die Kapitalwertmethode deutlich verbreiteter, benötigt weniger Eingabedaten und ist deutlich einfacher zu verstehen. In diesem Beitrag wird eine Methodik zur Bewertung von Wandelbarkeit in der Intralogistik vorgeschlagen, bei der die Kapitalwertmethode mit Methoden des Operations Research verbunden wird, um dem dynamischen Charakter von Wandelbarkeit Rechnung zu tragen. Daraus ergibt sich der Vorteil 
einer präzisen mathematischen Formulierung, die insbesondere auch einem analytischen Vorgehen zugänglich ist.

\section{$3 \quad$ Ein Verfahren zur Bewertung von Wandelbarkeit in der Intralogistik}

\section{1 Überblick}

Die Ausführungen in diesem Kapitel geben wesentliche Ergebnisse aus [NtH09] wieder. Dabei wird im Folgenden davon ausgegangen, dass der Zustand eines Materialflusssystems zu jedem Zeitpunkt über einen Vektor $\mathbf{x}(\mathbf{t})$ beschreibbar ist. Die Komponenten von $\mathbf{x}$ beschreiben das Materialflusssystem anhand der für die Wandelbarkeit relevanten Dimensionen Durchsatz, Fördergut und Layout. Beispielsweise kann $\mathbf{x}$ für ein Stetigfördersystem als Komponenten die Anzahl der Quellen und Senken in Stück, die Länge der Fördertechnik in Metern und die Anzahl verschiedener Ladehilfsmittel in Stück enthalten. In diesem Fall ist $\mathbf{x}$ also vierdimensional. Dabei ist darauf zu achten, dass $\mathbf{x}$ nicht mehr Komponenten enthält, als zur Beschreibung der Wandelbarkeit über den Lebenszyklus erforderlich sind. Weiterhin müssen alle Komponenten von $\mathbf{x}$ linear unabhängig sein. Falls also im erwähnten Beispiel die Anzahl der Quellen und Senken und die Länge der Fördertechnik (in beliebiger Weise, aber ausschließlich) abhängig sind vom Durchsatz, sind als Komponenten nur der Durchsatz sowie die Anzahl verschiedener Ladehilfsmittel zu wählen. Ändern sich nun letztere im gesamten Lebenszyklus nicht für alle zu betrachtenden Entwicklungen der Umwelt, ist die für die Wandelbarkeit relevante Größe des Materialflusssystems ausschließlich der Durchsatz; $\mathbf{x}$ wäre hier also eindimensional. Dabei ist es wichtig, den Bedarf an Systemgröße $\mathbf{x}_{\mathbf{B}}$ und das Angebot an Systemgröße $\mathbf{x}_{\mathbf{A}}$ zu unterscheiden. Im Beispiel entspricht der tatsächlich notwendige Durchsatz zu jedem Zeitpunkt $\mathbf{x}_{\mathbf{B}}$, wohingegen der maximal mögliche Durchsatz des Materialflusssystems zu jedem Zeitpunkt $\mathbf{x}_{\mathbf{A}}$ entspricht.

Abbildung 1 zeigt ein illustratives eindimensionales Beispiel, anhand dessen im Folgenden die vorgeschlagene Methodik zur Quantifizierung von Wandelbarkeit erläutert wird. Für dieses wachse der Bedarf an Größe des Materialflusssystems $\mathbf{x}_{\mathbf{B}}$ linear über die Zeit. Prinzipiell kann nun $\mathbf{x}_{\mathbf{A}}$ durch den Entscheidungsträger beliebig angepasst werden; für einen aussagekräftigen Vergleich verschiedener Systeme ist es jedoch erforderlich, einen für die gegebenen Parameter und den Verlauf von $\mathbf{x}_{\mathbf{B}}$ optimalen Verlauf von $\mathbf{x}_{\mathbf{A}}$ zu verwenden. Im Beispiel heißt das, dass es keinen anderen Verlauf von $\mathbf{x}_{\mathbf{A}}$ für das leicht und schwer wandelbare System gibt, der besser (d.h. wirtschaftlich günstiger) ist.

In Abbildung 1 sind die optimierten Verläufe von $\mathbf{x}_{\mathbf{A}}$ für ein leicht und ein schwerer wandelbares System gezeigt. $\mathbf{x}_{\mathbf{A}}$ folgt nun im Falle des leicht wandelbaren Systems dem Verlauf von $\mathbf{x}_{\mathbf{B}}$ enger als im Fall des schwer wandelbaren Systems. 


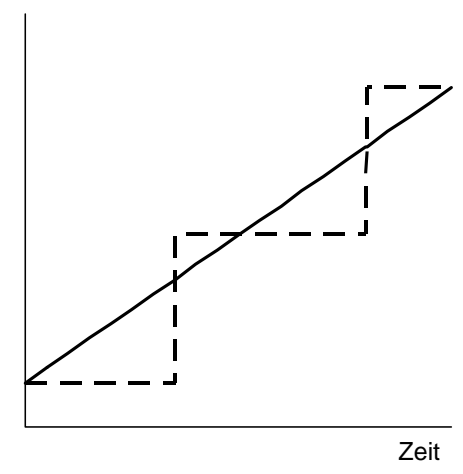

(a)

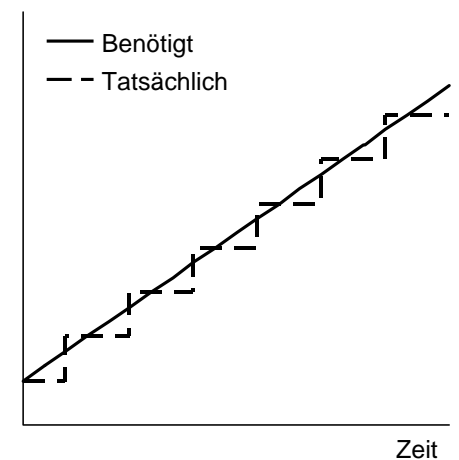

(b)

Abbildung 1: Verdeutlichung der Bedeutung von Wandelbarkeit, (a) - schwer wandelbares System, (b) - leicht wandelbares System

Dies ergibt sich aus der Optimierung des Systems hinsichtlich der Auszahlungen über den Lebenszyklus. Dabei lassen sich die relevanten Auszahlungen oft einer der folgenden Kategorien zuordnen:

- Auszahlungen in Folge von Betriebskosten O: Betriebskosten sind z.B. Energiekosten sowie Wartungs- und Instandhaltungskosten. Diese sind oft von der tatsächlichen Größe des Systems $\mathbf{x}_{\mathbf{A}}$ abhängig und werden in der Realität üblicherweise als jährliche Ausgaben bezogen auf die Investitionssumme angegeben. In aller Regel steigen Betriebskosten, wenn das Materialflusssystem größer wird. Um Betriebskosten zu minimieren, sollte also das Materialflusssystem immer so klein wie möglich sein.

- Auszahlungen in Folge von Überlastungskosten C: Falls Materialflusssysteme kleiner sind als benötigt, entstehen so genannte Überlastungskosten (engl. congestion costs). Diese können beispielsweise entgangenen Umsätzen Rechnung tragen oder durch manuelle Ersatztransporte verursacht werden. Überlastungskosten entstehen dann, wenn $\mathbf{x}_{\mathbf{A}}<\mathbf{x}_{\mathbf{B}}$. Um Überlastungskosten zu minimieren, sollte also das Materialflusssystem immer mindestens so groß sein wie notwendig.

- Auszahlungen in Folge von Investitionskosten I: Investitionskosten entstehen aufgrund von Erweiterungen des Materialflusssystems und sind im Allgemeinen abhängig von der Größe der Erweiterung $\mathbf{x}_{\mathbf{A}}$, die im Folgenden als $\mathbf{u}$ bezeichnet wird. Investitionskosten bestehen oft aus einem fixen und einem variablen Anteil: Der fixe Anteil $F I$ fällt unabhängig vom Betrag von u an, der variable Anteil hängt von $\mathbf{u}$ ab: $I(\boldsymbol{u})=F I+f(\boldsymbol{u})$. In erster Annäherung gilt, dass geringe fixe Investitionskosten kleine Anpassungen begünstigen.

Das sich so ergebende Optimierungsproblem ist verwandt mit so genannten Kapazitätserweiterungsproblemen aus dem Infrastruktur- und Produktionsbereich, die allerdings bisher nicht zur Untersuchung von Flexibilität oder Wandelbarkeit verwendet wurden (vgl. [Fre81], [Man61]). 


\subsection{Vorgehen}

Aufbauend auf diesen Vorüberlegungen wird im Folgenden eine quantitativ-monetäre Methodik zur Bewertung von Wandelbarkeit in der Intralogistik vorgeschlagen. Diese umfasst vier Schritte (vgl. Abbildung 2). Sie eignet sich besonders für die Intralogistik, berücksichtigt den gesamten Lebenszyklus des Materialflusssystems und erlaubt den Umgang mit einer unsicheren Zukunft. Darüber hinaus bietet die vorgeschlagene Methodik auch eine intuitiv zugängliche Erklärung für Wandelbarkeit, ist einfach erweiterbar und verursacht einen geringen Bewertungsaufwand.

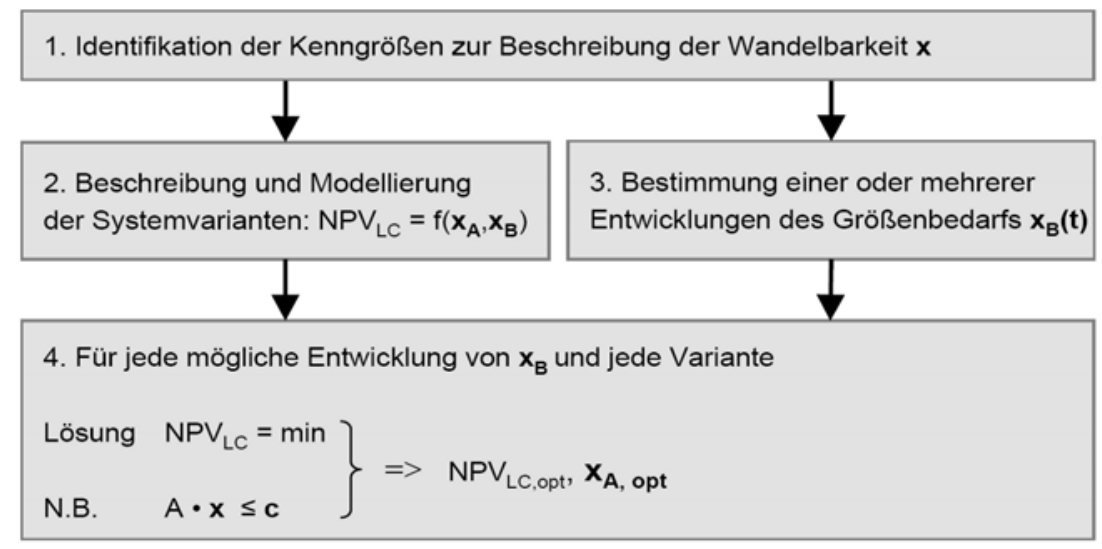

Abbildung 2: Vorgehen bei vorgeschlagener Methodik

Im ersten Schritt der Methodik wird eine geeignete Beschreibung des für die Wandelbarkeit relevanten Zustands des Materialflusssystems gesucht. Dies geschieht für alle Zeitpunkte mit Hilfe eines Vektors $\mathbf{x}(\mathbf{t})$ wie oben beschrieben. Im zweiten Schritt sind für jede betrachtete Systemvariante die durch die Wandelbarkeit beeinflussten Auszahlungen im Lebenszyklus zu modellieren. Für Materialflusssysteme sind in der Regel die Auszahlungen aufgrund von Betriebskosten, die Auszahlungen aufgrund von Engpasssituationen und die Auszahlungen aufgrund von Erweiterungs- und Anpassungsinvestitionen relevant. Im dritten Schritt wird nun die erwartete Entwicklung der Anforderungen an das System $\mathbf{x}_{B}$ über den Lebenszyklus prognostiziert. Dabei können beliebig viele mögliche Entwicklungen von $\mathbf{X}_{\mathbf{B}}$ betrachtet werden, die anschließend entsprechend ihrer Auftretenswahrscheinlichkeit gewichtet werden können. Im abschließenden vierten Schritt werden für jede zu betrachtende Entwicklung $X_{B}$ und jede Systemvariante die optimalen Anpassungsschritte über den Lebenszyklus bestimmt. Gleichzeitig ergibt sich daraus der Kapitalwert aller Auszahlungen im Lebenszyklus. Die Optimierung der Anpassungsschritte ist notwendig, um das bestmögliche Anpassungsprogramm jeder Systemvariante zu bestimmen und damit mögliche Bewertungsfehler durch einen Vergleich suboptimaler Anpassungsprogramme zu vermeiden. Die eigentliche Optimierung kann aufgrund der mathematischen Struktur des Problems mit Hilfe einer dynamischen Optimierung durchgeführt werden. Diese beruht auf dem Bellman'schen Optimalitätsprinzip, das stets die Bestimmung des globalen Optimums erlaubt. 
Das Optimierungsproblem kann folgendermaßen formuliert werden:

$$
N P V_{L C}=\sum_{j=1}^{N} e^{-r_{j} t_{j}} \cdot\left[I\left(u_{j}\right)+C\left(x_{A, j}, x_{B, j}\right)+O\left(x_{A, j}\right)\right] \rightarrow M I N
$$

unter der dynamischen Nebenbedingung:

$$
x_{A, j}=f\left(x_{A, j-1}, u_{j}\right)=x_{A, j-1}+u_{j}
$$

und mit den statischen Nebenbedingungen:

$$
\begin{aligned}
& x_{A, 0}=\text { gegeben } \\
& x_{A, j} \in \Xi_{j} \\
& u_{j} \in \Omega_{j}\left(x_{A, j-1}\right) \\
& \Xi_{j}=\left[0, \max _{j}\left(x_{B, j}\right)\right] \\
& \Omega_{j}\left(x_{A, j-1}\right)=\left[-x_{A, j-1}, \max _{j}\left(x_{B, j}\right)-x_{A, j-1}\right]
\end{aligned}
$$

Leicht wandelbare Systeme zeigen nun im Optimum kleinere und dafür häufigere Anpassungen an die Anforderungen $\mathbf{X}_{\mathbf{B}}$ als schwer wandelbare Systeme (vgl. Abbildung 1). Dadurch ergeben sich Vorteile hinsichtlich der Auszahlungen für den laufenden Betrieb, für die Anpassungs- und Erweiterungsinvestitionen und für die Auszahlungen in Folge von Unterdeckungen. Im Fall einer unsicheren Zukunft ergibt sich ein zusätzlicher Vorteil, da der Informationsgewinn im Zeitverlauf besser genutzt werden kann. Die vorgeschlagene Methodik ist nicht auf die Untersuchung selbstorganisierter Materialflusssysteme beschränkt, sondern kann auf die Quantifizierung einer von mit beliebigen Mitteln ermöglichten Wandelbarkeit in der Intralogistik übertragen werden.

Wesentlich für die Wandelbarkeit eines Materialflusssystems ist dabei oft die Höhe der fixen Investitionskosten. Damit ist der Teil der Investitionskosten gemeint, der unabhängig von der Größe der Erweiterung oder Anpassung anfällt. Er entsteht in der Intralogistik unter anderem durch Abnahmen und Tests im Rahmen der Inbetriebnahme.

\subsection{Der Wert der Flexibilität}

Auf dieser Basis lässt sich ein Wert der Wandelbarkeit W definieren, der sich für Grenzfälle analytisch berechnen lässt:

$W=\frac{N P V_{\text {starr }}-N P V}{N P V_{\text {starr }}}$

Hierfür wird das gegebene System mit einem starren System verglichen, das im Lebenszyklus nicht angepasst wird, ansonsten aber die gleichen Eigenschaften wie 
das System hat. W nimmt für ein gegebenes System und eine gegebene Entwicklung der Anforderungen einen Wert zwischen 0 und 1 an. Ein Wert von 0 bedeutet, dass Wandelbarkeit für das System und die jeweiligen Anforderungen keine Rolle spielt der Kapitalwert der Auszahlungen im Lebenszyklus für das betrachtete und das starre System sind gleich. Ein Wert von 1 bedeutet dagegen einen maximalen Wert der Wandelbarkeit. In diesem Fall entfallen alle Auszahlungen im Lebenszyklus für das wandelbare System. Reale Systeme nehmen somit stets einen Wert zwischen 0 und 1 an.

Der maximal mögliche Wert der Wandelbarkeit $W_{\max }$ kann für einige Systeme auch analytisch und ohne Durchführung der dynamischen Optimierung berechnet werden. Beispielsweise ist dies möglich für ein eindimensionales Materialflusssystem mit linearer Bedarfsentwicklung und linearen Kostenfunktionen. Dabei wird davon ausgegangen, dass eine erhöhte Wandel-barkeit eines Systems gleichbedeutend mit geringen fixen Kosten jeder Änderung $(F I)$ ist.

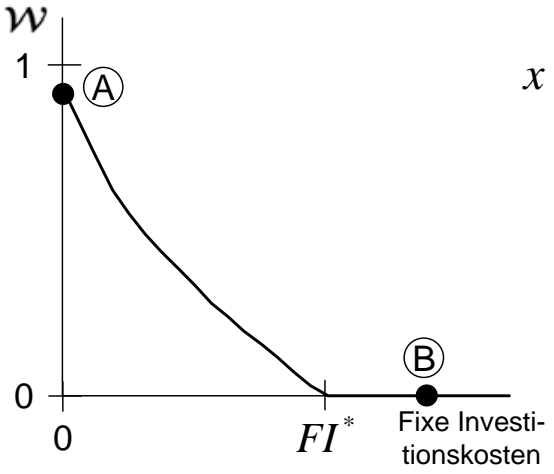

(a)

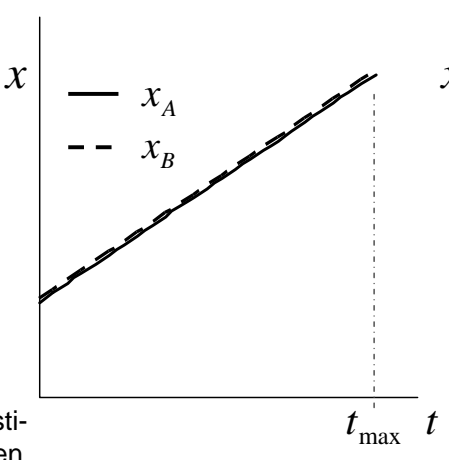

(b)

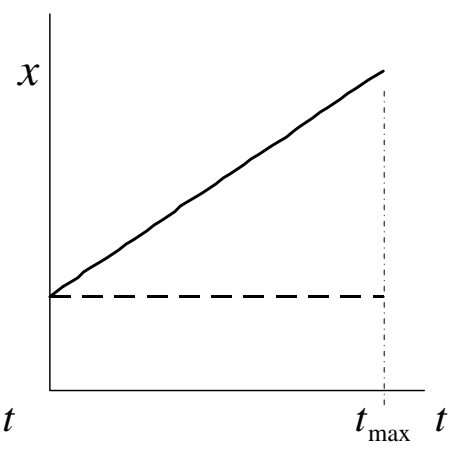

(c)

Abbildung 3: Analytische Berechnung des Werts der Wandelbarkeit. (a) - Wert der Wandelbarkeit über fixen Investitionskosten, (b) - tatsächliche und benötigte Systemgröße an Punkt A, (c) - tatsächliche und benötigte Systemgröße an Punkt $B$.

In Abbildung 3 werden die wesentlichen Zusammenhänge dargestellt: Grundsätzlich hängt die Wandelbarkeit des Systems wie beschrieben von den fixen Investitionskosten FI ab. Der Fall verschwindender fixer Investitionskosten entspricht gleichzeitig dem maximalen Wert der Wandelbarkeit $W_{\max }$. Dabei sinkt der Wert der Wandelbarkeit gegenüber dem starren System mit steigenden Fixkosten je Änderung (Abbildung 3a). Falls keinerlei Investitionsfixkosten für eine Änderung anfallen, ist es optimal, das System zu jedem Zeitpunkt an die Anforderungen anzupassen (Abbildung 3b). Falls dagegen die Fixkosten je Änderung einen bestimmten Wert überschreiten ( $\left.\mathrm{FI}>\mathrm{FI}^{\star}\right)$ ist es wirtschaftlicher, keine Anpassung durchzuführen und die Überlastungskosten $C$ in Kauf zu nehmen (Abbildung 3c). ei nun das System durch folgende Zusammenhänge charakterisiert:

$$
N P V_{L C}=\sum_{j=1}^{N} e^{-r_{j} \cdot t_{j}} \cdot[I+C+O]
$$

mit den Kostenfunktionen 


$$
\begin{gathered}
I=\left\{\begin{array}{c}
F I+u_{j} \cdot \sigma, \quad \text { falls } u_{j}>0 \\
0, \quad \text { falls } u_{j}=0
\end{array}\right. \\
O=\beta \cdot I_{\text {variabel }}\left(x_{A, j}\right) \cdot \Delta t=\beta \cdot x_{A, j} \cdot \sigma \cdot \Delta t \\
C=\left\{\begin{array}{c}
\delta \cdot\left(x_{B, j}-x_{A, j}\right) \cdot \Delta t \quad \text { falls } x_{A, j}<x_{B, j} \\
0, \quad \text { falls } \quad x_{A, j} \geq x_{B, j}
\end{array}\right.
\end{gathered}
$$

Dann kann der Wert der Wandelbarkeit analytisch über Integration und Umformung bestimmt werden zu

$$
W=\frac{e^{-r \cdot t_{\max }} h\left(\sigma r+\beta \sigma t_{\max } r+\beta \sigma-\delta t_{\max } r-\delta\right)+h(\delta-\sigma r-\beta \sigma)}{\delta h+\beta \sigma g r-e^{-r \cdot t_{\max }}\left(\delta h t_{\max } r+\delta h+\beta \sigma g r\right)}
$$

W kann also geschlossen in Abhängigkeit von 7 Parametern angegeben werden. Diese sind mit ihren Einheiten in Tabelle 1 dargestellt. So kann für jede Problemstellung mit bekanntem linearem Wachstum vorab der maximale Wert der Wandelbarkeit berechnet werden. Hierüber lässt sich abschätzen, ob sich der Einsatz wandelbarer Materialflusssysteme lohnt. So ist z.B. $W_{\max }=W(F I=0)=0$, falls $h=0$.

Tabelle 1: Parameter von $\mathrm{W}$ im analysierten linearen Beispiel

\begin{tabular}{lll}
\hline Parameter & Bedeutung & Einheit \\
\hline$g$ & $x_{B}(t=0)=x_{A}(t=0)$ & Größe \\
$h$ & Wachstum von $x_{B}$ & Größe/Zeit \\
$r$ & Zinssatz & $1 /$ Zeit \\
$t_{\max }$ & Betrachtungszeitraum & Zeit \\
$\beta$ & Betriebskostensatz & $1 /$ Zeit \\
$\delta$ & Überlastungskosten & $€ /$ Göße/Zeit \\
$\sigma$ & Variable Investitionskosten & $€ G r o ̈ ß e$ \\
\hline
\end{tabular}

\subsection{Wandelbarkeit und Entscheidung unter Unsicherheit}

Anders als bisher dargestellt kennt ein Entscheider die zukünftige Entwicklung der Anforderungen $\mathbf{x}_{\mathbf{B}}$ in aller Regel nicht. In diesem Fall hat Wandelbarkeit einen zusätzlichen Wert, da für ein wandelbares System kleinere Anpassungen vorgenommen werden als für ein weniger wandelbares System. Dies führt zu häufigeren und im Durchschnitt späteren Anpassungen des Systems, wodurch bei jeder Anpassungsentscheidung mehr Informationen berücksichtigt werden können als bei einem weniger wandelbaren System. Dies stellt gewissermaßen eine Versicherung gegen Unsicherheit dar. Diese kann unter gewissen Annahmen 
ebenfalls quantifiziert werden. Es lässt sich zeigen, dass sich hierfür ein zusätzlicher Nutzen der Wandelbarkeit ergibt.

\section{$4 \quad$ Beispiel: Ein fahrerloses Transportsystem}

Die Methodik zur Quantifizierung von Wandelbarkeit in der Intralogistik wurde bereits in mehreren praxisnahen Beispielen überprüft: Beispielsweise untersuchen [Kle09] und [Mod09] in zwei Arbeiten den Nutzen von Wandelbarkeit in Distributionszentren bzw. einem fahrerlosen Transportsystem in der Automobilindustrie. Im Folgenden soll ein kurzer Einblick in die praktische Anwendung der Methodik gegeben werden, die [NtH09] entnommen wurde. Dabei wird grundsätzlich ein schwer wandelbares („starres“) System mit einem leicht wandelbaren System verglichen.

Betrachtet wird ein fahrerloses Transportsystem (FTS) mit einer Lebensdauer von 20 Jahren. Die Kapitalkosten sollen $10 \%$ betragen. Das FTS wird in einer Fertigung eingesetzt, bei der die Produktionsmenge des hergestellten Produkts über die Lebensdauer linear von 0 auf 1.000 Stück pro Tag anwachse. Hierbei kann ein Fahrzeug des FTS 100 Stück des Produkts transportieren. Die Betriebskosten des FTS seien 15\% der variablen Investitionskosten. Falls das FTS zu klein ist, fallen Mehrkosten von 5 Geldeinheiten je manuell transportiertem Stück an. Die variablen Investitionskosten sollen 100.000 Geldeinheiten je Fahrzeug betragen, die fixen Investitionskosten seien 100.000 Geldeinheiten für das starre und 30.000 Geldeinheiten für das leicht wandelbare System. Das Problem wird mit Hilfe der vorgestellten Methodik gelöst, wobei die Periodenlänge ein Jahr betrage.

Zunächst sind die relevanten Kenngrößen zur Beschreibung von Wandelbarkeit zu bestimmen. Für das gegebene Beispiel seien Layout und Durchsatz der eingesetzten Fördertechnik direkt abhängig von der Produktionskapazität $x$. Das Fördergut sei konstant für den gesamten Betrachtungszeitraum. Die einzige zur Beschreibung der notwendigen Transportleistung relevante Größe für das System ist damit $x$. Das dargestellte Problem lässt sich damit folgendermaßen beschreiben:

$$
\begin{aligned}
& N P V_{L C}=\sum_{j=1}^{21} e^{-0,1 \cdot(j-1)} \cdot\left[I\left(u_{j}\right)+C\left(x_{A, j}, x_{B, j}\right)+O\left(x_{A, j}\right)\right] \\
& I_{\text {starr }}\left(u_{j}\right)=\left\{\begin{array}{cc}
1.000 \cdot u_{j}+100.000 & \forall\left(u_{j}>0\right) \\
0 & \forall\left(u_{j}=0\right) \\
100.000 & \forall\left(u_{j}<0\right)
\end{array}\right. \\
& I_{\text {wamdelbar }}\left(u_{j}\right)=\left\{\begin{array}{cc}
1.000 \cdot u_{j}+30.0000 & \forall\left(u_{j}>0\right) \\
0 & \forall\left(u_{j}=0\right) \\
30.000 & \forall\left(u_{j}<0\right)
\end{array}\right. \\
& C\left(x_{A, j}, x_{B, j}\right)=\left\{\begin{array}{cc}
1.000 \cdot\left(x_{B, j}-x_{A, j}\right) & \forall\left(x_{B, j}>x_{A, j}\right) \\
0 & \forall\left(x_{B, j} \leq x_{A, j}\right)
\end{array}\right.
\end{aligned}
$$


Dieses Problem lässt sich wie beschrieben über eine dynamische Optimierung lösen. Die Ergebnisse aller Berechnungen sind in Tabelle 2 dargestellt.

Tabelle 2: Ergebnisse des analysierten Beispiels

\begin{tabular}{llll}
\hline (Geldeinheiten) & Starr & Wandelbar & Delta \\
\hline Betrieb & 489.981 & 483.034 & 6.948 \\
Investition (fix) & 198.202 & 124.997 & 73.205 \\
Investition (var.) & 421.063 & 416.656 & 4.408 \\
Ersatztransporte & 130.535 & 21.012 & 109.523 \\
\hline Summe & 1.239 .782 & 1.045 .698 & 194.084 \\
\hline
\end{tabular}

Für das betrachtete Beispiel ist das wandelbare System bezüglich der kumulierten Auszahlungen etwa $16 \%$ günstiger als das konventionelle System. Es ist beachtenswert, dass die Einsparung von fixen Investitionskosten nur knapp 40\% des Unterschieds zwischen beiden Systemen ausmacht, obwohl dies der einzige Unterschied zwischen den Systemen ist (Tabelle 2). Zusätzlich entstehen geringere Auszahlungen zur Aufrechterhaltung des Betriebs und aufgrund zu geringer Transportleistung des FTS, da sowohl die durchschnittliche Größe des FTS als auch der Bedarf für manuelle Ersatztransporte in Folge von Unterdeckungen für das wandelbare System geringer sind als die des starren Systems. Der Unterschied der variablen Investitionskosten - trotz einer am Ende gleichen Größe - ergibt sich durch die späteren Investitionszeitpunkte im Falle des selbstorganisierten Systems. Im gewählten Beispiel verursacht das selbstorganisierte System damit in jeder Komponente geringere kumulierte Auszahlungen. Das ist im Allgemeinen nicht der Fall, zeigt aber, dass ein reiner Vergleich der eigentlichen Investitionskosten zu einer deutlichen Unterschätzung der Vorteile flexibler Systeme führen kann.

\section{$5 \quad$ Zusammenfassung und Ausblick}

Die im Rahmen dieses Artikels vorgestellte Methodik ist dazu geeignet, Wandelbarkeit in der Intralogistik allgemeingültig zu beurteilen. Sie bietet insgesamt einen anhand von praktischen Beispielen überprüften Rahmen zur strukturierten Auswertung von Daten realer Materialflusssysteme. Darüber hinaus fördert sie das Verständnis von Wandelbarkeit in der Intralogistik, da sie ein einfach zugängliches Erklärungsmodell für Vorteile durch Wandelbarkeit liefert.

Sinnvolle Linien für die Weiterentwicklung der Methodik umfassen neben der analytischen Betrachtung nicht-linearer Entwicklungen des Bedarfs insbesondere auch die weitere Untersuchung des Zusammenhangs zwischen unbekannter Zukunft und Wandelbarkeit interessant. Weiterer Forschungsbedarf ergibt sich im Zusammenhang mit einer stochastischen Bedarfsentwicklung; so kann die vorgeschlagene Methodik mit einer Monte-Carlo-Simulation oder der Szenariotechnik auszuwerten. 


\section{Literatur}

[BtH07] Bullinger, H.-J.; ten Hompel, M. (Hrsg.): Internet der Dinge. Berlin Heidelberg New York: Springer, 2007.

[Fre81] Freidenfels, J.: Capacity Expansion: Analysis of Simple Models with Applications. New York: Elsevier, 1981.

[GCK08a] Günthner, W.; Chisu, R.; Kuzmany, F.: Das "Internet der Dinge" - Teil I: Intelligent verteilt. In: F+H, (7-8):422-425, 2008.

[GCK08b] Günthner, W.; Chisu, R.; Kuzmany, F.: Das "Internet der Dinge" - Teil II: Steuern ohne Hierarchie. In: F+H, (9):494-497, 2008.

[GCK08c] Günthner, W.; Chisu, R.; Kuzmany, F.: Das "Internet der Dinge" - Teil III: Zukunftstechnologie mit Kostenvorteil. In: F+H, (10):556-558, 2008.

[GHW02] Günthner, W.; Heinecker, M.; Wilke, M.: Materialflusssysteme für wandelbare Fabrikstrukturen. In: Industrie Management, 18(5):8-10, 2002.

[Kle09] Klein-Hitpass, U.: Bewertung des Nutzens selbstorganisierter Materialflusssysteme in Distributionszentren. Dortmund: Diplomarbeit, Technische Universität Dortmund, 2009.

[Man61] Manne, S. A.: Capacity expansion and probabilistic growth. In: Econometrica, 29(4):632-649, 1961.

[May09] Mayer H. S.: Development of a completely decentralized control system for modular continuous conveyors. Karlsruhe: Dissertation, Universität Karlsruhe (TH), 2009.

[Mod09] Modrok, C.: Bewertung des Nutzens selbstorganisierter fahrerloser Transportsysteme in der Automobilindustrie. Dortmund: Diplomarbeit, Technische Universität Dortmund, 2009.

[Möl08] Möller, N.: Bestimmung der Wirtschaftlichkeit wandlungsfähiger Produktionssysteme. München: Dissertation, TU München, 2008.

[NtH09] Nopper, J.R.; ten Hompel, M.: Methodik zur Bewertung von Effizienzgewinnen durch erhöhte Flexibilität selbstorganisierter Systeme in der Intralogistik. In: Logistics Journal, (7), 2009.

[Sla83] Slack, N.: Flexibility as a manufacturing objective. In: International Journal of Operations \& Production Management, 3(3):4-13, 1983.

[SeS90] Sethi, K.; Sethi, P. S.: Flexibility in Manufacturing: A Survey. The International Journal of Flexible Manufacturing Systems, (2):289-328, 1990.

[tHFR08] ten Hompel, M.; Follert, G.; Roidl, M.: Künstliche Intelligenz im Internet der Dinge: Die Zukunft der Materialflusssteuerung mit autonomen Agenten. In: Jahrbuch Logistik 2008, Seiten 24-29. Korschenbroich: free Beratung $\mathrm{GmbH}, 2008$. 